\title{
Doppler waveform patterns and reference ranges of fetal renal artery blood flow indices in normal Thai fetuses during the second trimester
}

Surachai Ponglopisit ${ }^{\mathrm{a}}$, Tharangrut Hanprasertpong ${ }^{\mathrm{a}}$, Alan Geater ${ }^{\mathrm{b}}$, Chusana Petpichetchian ${ }^{\mathrm{a}}$, Ounjai Kor-anantakula, Roengsak Leetanaporna, Thitima Suntharasaja, Chitkasaem Suwanrath ${ }^{\mathrm{a}}$, Ninlapa Pruksanusak ${ }^{\mathrm{a}}$, Savitree Pranpanus ${ }^{\mathrm{a}}$, Walailak Jitpiboon ${ }^{\mathrm{a}}$

${ }^{a}$ Department of Obstetrics and Gynaecology, Faculty of Medicine, Prince of Songkla University, Hatyai, Songkla 90110, Thailand

${ }^{b}$ Epidemiology Unit, Faculty of Medicine, Prince of Songkla University, Hatyai, Songkla 90110, Thailand

\begin{abstract}
Background: Fetal hemodynamics can be assessed by Doppler ultrasonography, but the normal Doppler waveform pattern and reference range of fetal renal artery blood flow indices in normal Thai fetuses during the second trimester have not been studied.

Objectives: To study the pattern and establish the normal reference range of fetal renal artery blood flow indices in the normal Thai fetus during the second trimester.

Materials and methods: This cross-sectional descriptive study included 512 normal singleton pregnant women, gestational age between $14^{(+0)}$ and $28^{(+6)}$ weeks. Ultrasonography was performed to assess the Doppler waveform pattern and estimate the normal reference range of fetal renal artery blood flow indices. All fetuses were delivered at term with normal outcomes at birth. The mean and $95^{\text {th }}$ and $5^{\text {th }}$ percentiles of the Doppler indices for each gestational week were estimated.

Results: Doppler patterns with absence of diastolic flow in the entire cardiac cycle (type I), and absence of diastolic flow at the end of cardiac cycle (type II) were presented in about $10.4 \%$ of normal fetuses during the second trimester of pregnancy. Values for pulsatility index, peak systolic velocity, systolic-to-diastolic ratio, and resistance index (with absent end-diastolic velocity (AEDV) removed) each increased significantly with gestational age. The increase in end-diastolic velocity (with AEDV removed) with gestational age was not significant.

Conclusion: These normative data could serve as a basis for evaluation of the fetal renal artery blood flow, which should be of benefit for pregnancy management, especially in situations that interfere with fetal renal perfusion.
\end{abstract}

Keywords: Renal artery, second trimester

Doppler ultrasonography is a modality that has been used for assessment of fetal hemodynamics. The ultrasonography is represented as both qualitative findings and quantitative parameters. The qualitative findings describe the pattern of blood flow such as present, absent and reverse flow, whereas the quantitative parameters are considered as Doppler indices such as the systolic-to-diastolic ratio (S/D), pulsatility index (PI), and resistance index (RI).

Correspondence to: Tharangrut Hanprasertpong, Department of Obstetrics and Gynaecology, Faculty of Medicine, Prince of Songkla University, Songkhla 90110, Thailand.

E-mail: htharang@medicine.psu.ac.th
The kidney is an important organ for intrauterine fetal life. Several reports have described the adaptation of vascular anatomy and vasomotor regulation in the renal artery in pathological situations such as hypoxic stress in intrauterine growth restriction and the inflammation process of chorioamnionitis $[1,2]$. However, the Doppler waveform pattern and normal reference range of fetal renal artery blood flow indices in normal Thai fetuses during second trimester have not been reported. The aim of this study was to show the normal pattern and establish the normal reference range of fetal renal artery blood flow in the Thai fetus during the second trimester. 


\section{Materials and methods}

This was a cross-sectional descriptive study performed at the Maternal-Fetal Medicine Unit, Department of Obstetrics and Gynecology, Faculty of Medicine, Prince of Songkla University, Hat Yai, Thailand, between July 2012 and June 2013. This study was approved by the Ethics Committee of the Faculty of Medicine, Prince of Songkla University (approval number 55-319-12-3-3). Normal singleton pregnant women with a gestational age between $14^{(+0)}$ and $28^{(+6)}$ weeks who attended the antenatal care clinic at our hospital were recruited into our study. The gestational age of all participants was documented by the last menstrual period (LMP) and confirmed by ultrasonographic parameters since the first trimester of pregnancy. The exclusion criteria were (1) presentation with fetal malformations or abnormal fetal karyotype, (2) subsequent abnormal fetal growth in the index pregnancy including fetal growth restriction and fetal macrosomia, (3) presentation with an abnormal amount of amniotic fluid, (4) presentation with maternal medical disease such as hypertension, autoimmune disorder, and (5) subsequent obstetric complication in the index pregnancy such as preterm or post-term delivery.

All participants underwent 2-dimensional (2D) real-time ultrasonography conducted by a maternal fetal medicine fellow (PS) who had experience of renal artery Doppler measurement in more than 30 cases before this study using a Voluson E8 (GE Medical Systems, Kretztechnik, Zipf, Austria) with a $2-5 \mathrm{MHz}$ curvilinear probe. First, the number of fetuses, gestational age, amount of amniotic fluid, and standard structural screening were evaluated. Then the fetal kidneys were magnified and displayed in a longitudinal plane. A color Doppler ultrasound with a $60 \mathrm{~Hz}$ highpass filter was initiated. The fetal renal artery laterally branches from the abdominal aorta and extends directly toward to the kidney hilus. The transducer was adjusted so that the abdominal aorta lay parallel to the ultrasonographic beam. In this position, the insonation angle was as close to $0^{\circ}$ as possible. The sample volume was adjusted to $1-3 \mathrm{~mm}$ depending on the size of the fetal renal artery and placed approximately $1 \mathrm{~cm}$ after it branches off its origin from the abdominal aorta. All examinations were performed during absence of fetal movement or fetal breathing. PI, RI, S/D, peak systolic velocity (PSV) and enddiastolic velocity (EDV) measurements were performed and the presence or absence of end diastolic blood flow assessed. At least three consecutive waveforms from each participant were calculated by an automatic waveform analysis function integrated into the ultrasonographic device.

All participants were followed for maternal and neonatal outcomes until delivery. Only participants free of maternal medical complication, with normal neonatal birthweight and with no fetal anomalies were collected for statistical analysis.

\section{Statistical analysis}

The distributions of each Doppler index according to gestational age were examined using scatter plots. Appropriate transformations were then applied to each index to achieve an approximately symmetrical distribution for each gestational age. PI and PSV were log transformed, SD log-log transformed, EDV log transformed after removing fetuses with absent end-diastolic velocity (AEDV), and RI square root transformed after removing fetuses with AEDV. Each of the transformed indices was then regressed against gestational age in days using linear regression models to obtain the estimated mean value for each age, and the residuals of the model checked for near normality and the standard deviation (SD) of the residuals computed. Values of the $95^{\text {th }}$ and $5^{\text {th }}$ percentiles of each index were then estimated using the estimated means $\pm 1.64 *$ SD and the transformed data together with the mean and $95^{\text {th }}$ and $5^{\text {th }}$ percentiles were plotted against gestational age. The regression equations for each Doppler index were then used to tabulate the estimated mean and $95^{\text {th }}$ and $5^{\text {th }}$ percentiles for each day of gestational age. All analyses were conducted using STATA version 10 (StataCorp, College Stations, TX, USA).

\section{Results}

All 512 pregnant women were included for final analysis for a Doppler profile of the fetal renal artery. Table 1 shows the basic population characteristic. The mean maternal age was 31.39 (SD 6.11) years (range 15-47 years). About one third (188/512, 36.7\%) were nulliparous. Mean gestational age at delivery was 38.22 (SD 1.19) weeks and mean fetal birth weight was 3187.35 (SD 388.94) g. Figure 1 shows the 3 types of normal fetal renal artery Doppler pattern found in normal fetuses during the second trimester of pregnancy, described as absence of diastolic flow in the entire cardiac cycle (type I), absence of diastolic flow at the end of cardiac cycle (type II), presence of diastolic flow in the entire cardiac cycle (type III). Our study found that type I and type II presented in about $10.4 \%$ of normal fetuses during second trimester of pregnancy. 
Table 1. The population characteristics

\begin{tabular}{lll}
\hline Variable & Number & Percentage \\
\hline $\begin{array}{l}\text { Mother' age (years) } \\
\leq 19\end{array}$ & 25 & \\
$20-34$ & 346 & 4.9 \\
$\geq 35$ & 141 & 67.6 \\
Gravida & & 27.5 \\
1 & 194 & 37.9 \\
2 & 158 & 30.9 \\
3 & 104 & 20.3 \\
4 & 36 & 7.0 \\
$\geq 5$ & 20 & 3.9 \\
Para & & \\
1 & 233 & 45.5 \\
2 & 186 & 36.3 \\
$\geq 3$ & 65 & 12.7 \\
\hline
\end{tabular}
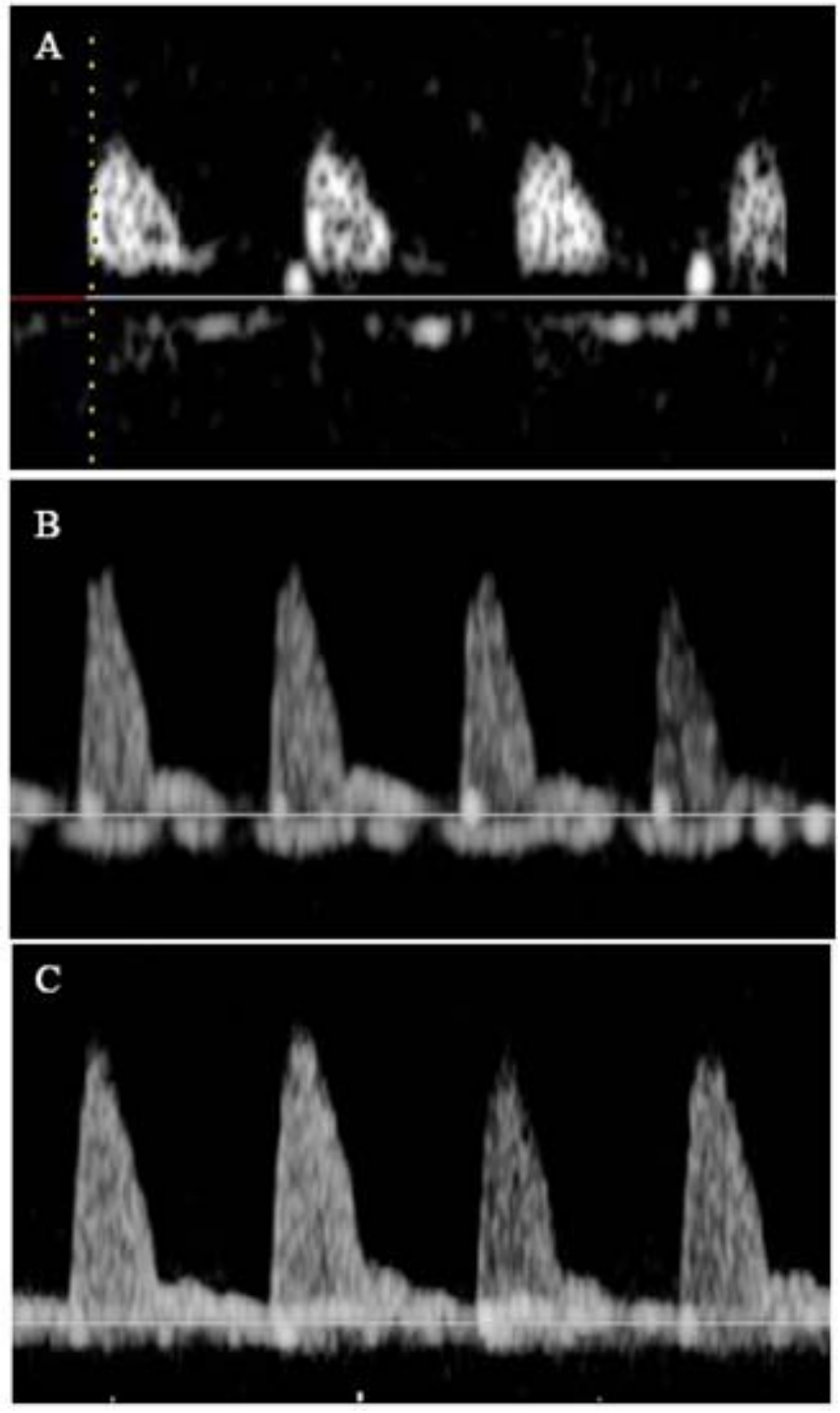

Figure 1. Flow-velocity waveforms in the fetal renal artery. A: Type I (absence of diastolic flow in the entire cardiac cycle) at 15 weeks (systolic waveform is visualized). B: Type II (absence of diastolic flow at the end of cardiac cycle) at 20 weeks (the systolic waveform is present and the diastolic waveform is absent). C: Type III at 24 weeks (systolic waveform is present and the diastolic waveform progresses into the following systolic waveform). 
The distributions of Doppler indices according to gestational age from $14^{(+0)}$ and $28^{(+6)}$ weeks are shown in Figure 2. Transformations achieved a more symmetrical distribution of each index by gestational age (Figure 3). The predicted mean and estimated $95^{\text {th }}$ and $5^{\text {th }}$ percentile lines for each index are also shown on Figure 3. Table 2 summarizes the predicted means and estimated $95^{\text {th }}$ and $5^{\text {th }}$ percentiles of each Doppler index for each week of gestation age between 14 and 28 weeks achieved after backtransformation to the original metric. Values of PI, PSV, S/D and RI (with AEDV removed) each increased significantly with gestational age. The increase in EDV (with AEDV removed) with gestational age was not statistically significant.
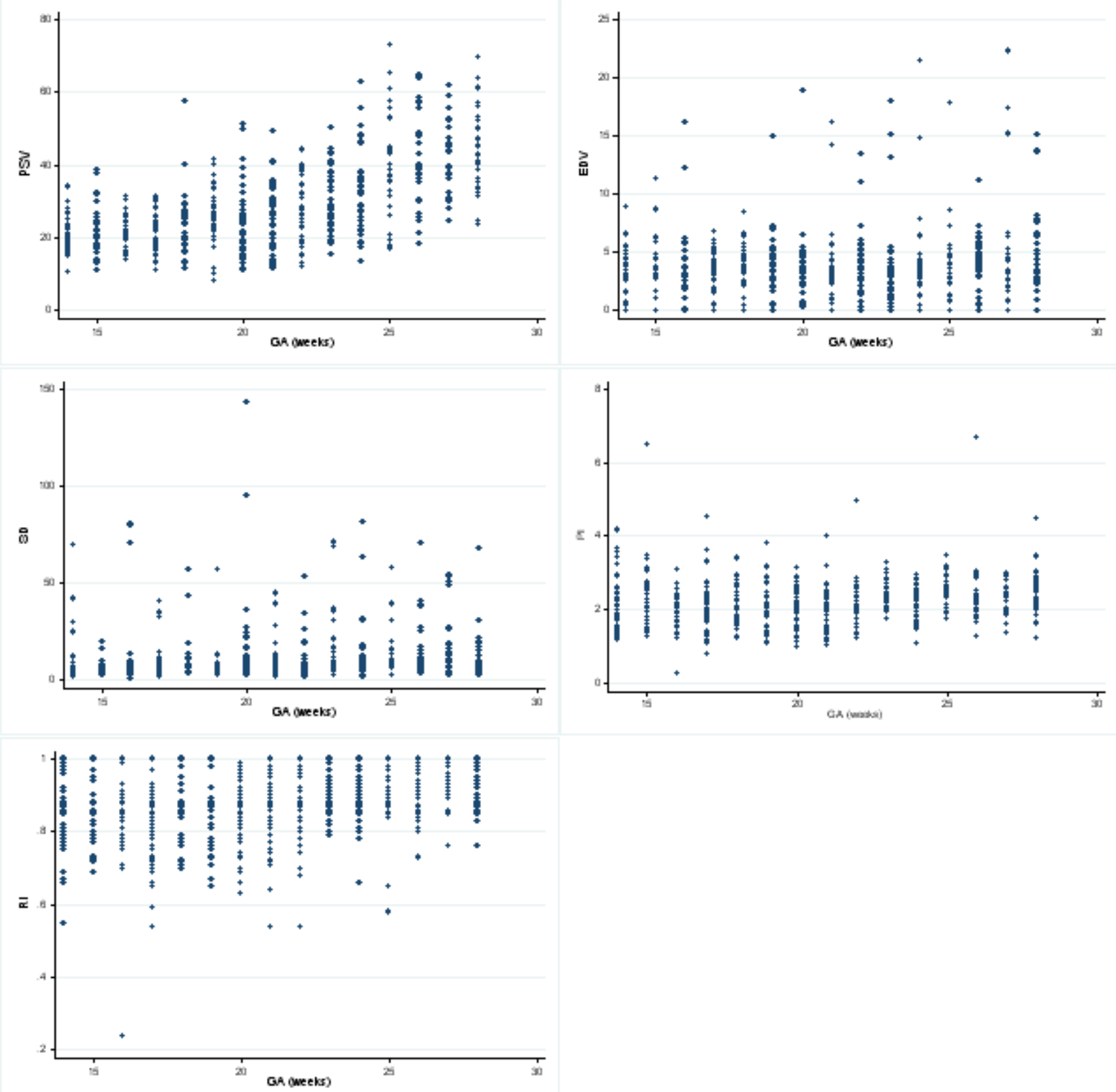

Figure 2. Scatter plots Doppler indices of the renal artery according to gestational age 

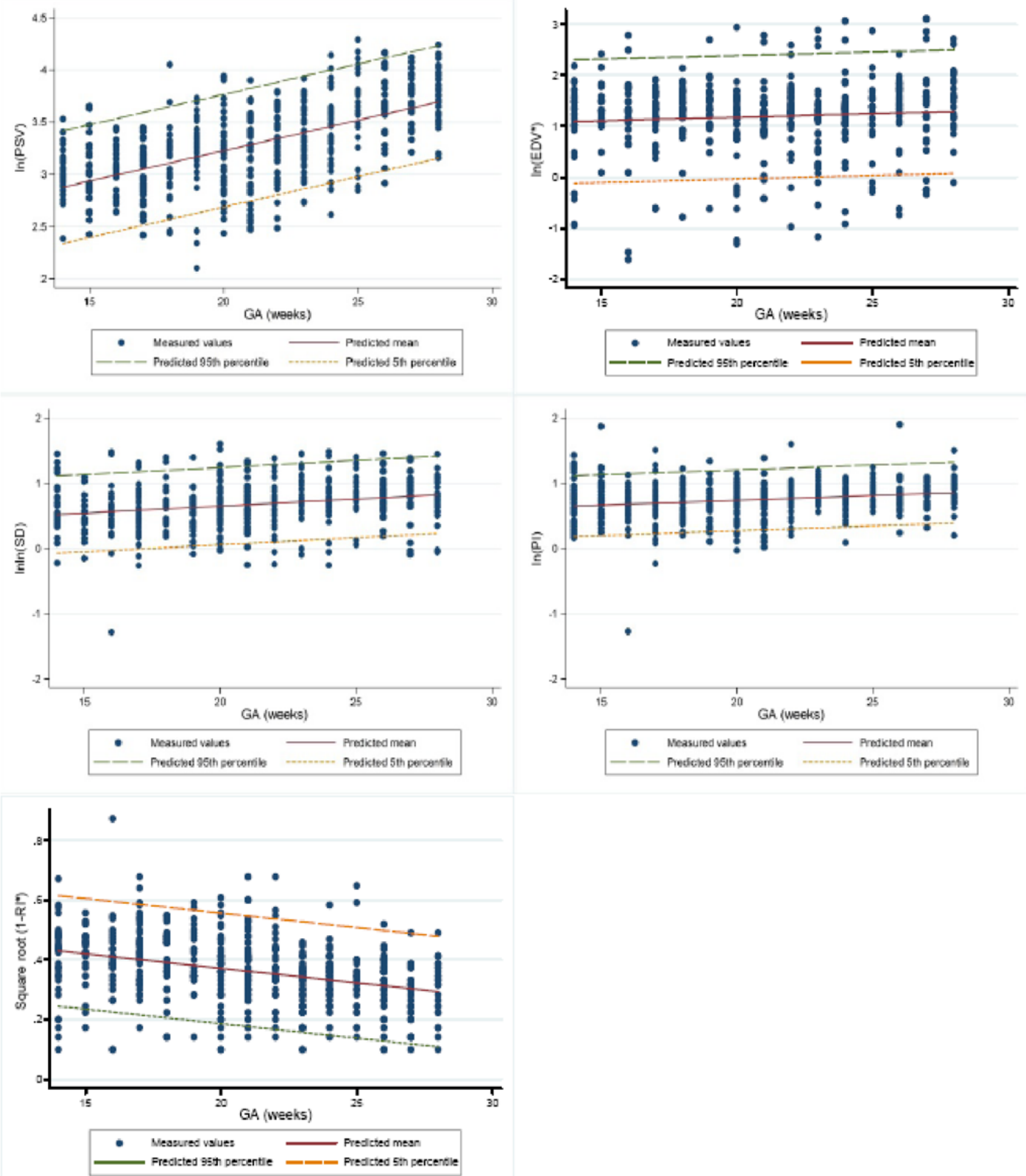

Figure 3. Scatter plots of the transformed Doppler indices of the renal artery according to gestational age, together with predicted mean and estimated $95^{\text {th }}$ and $5^{\text {th }}$ percentiles 


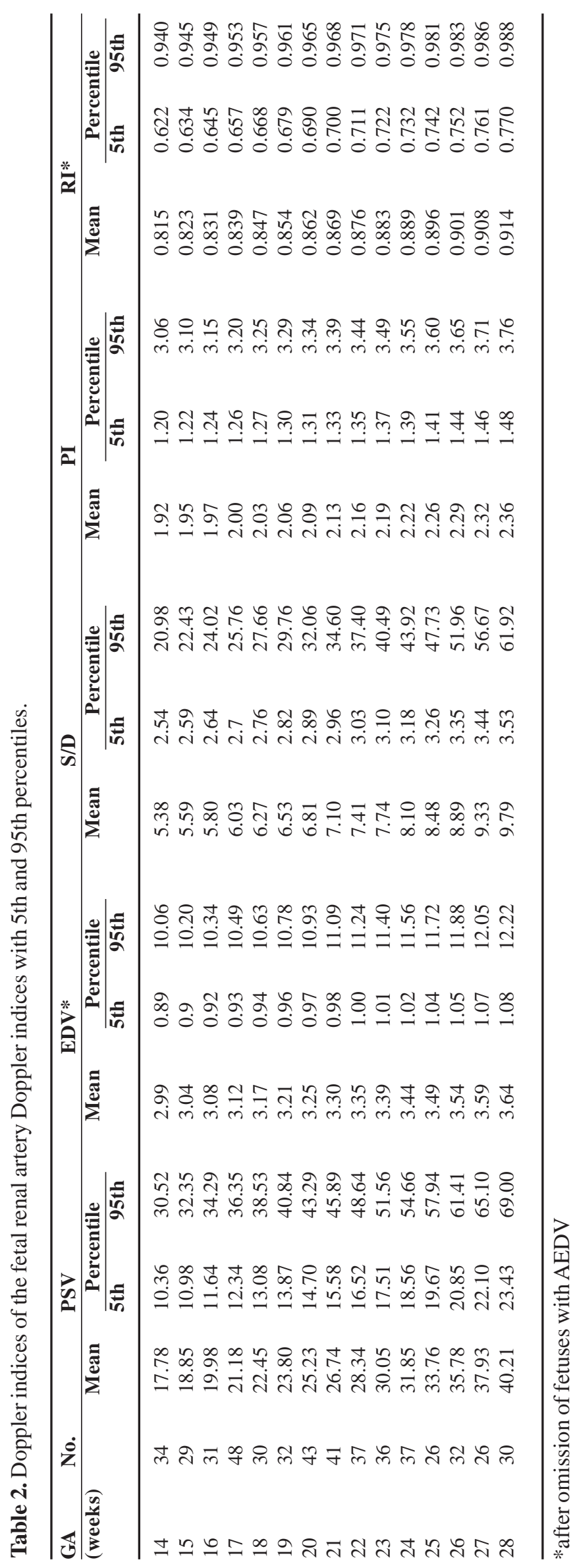




\section{Discussion}

To our knowledge, this is the first study to completely demonstrate both qualitative and quantitative fetal renal artery Doppler flow during the second trimester of pregnancy in Thai fetuses. Moreover, the fetal and maternal outcomes were followed until delivery to confirm normal pregnancy outcome. A few previous studies have presented normal values of renal artery Doppler flow [3-9], but the current study is the first to include fetuses in the early second trimester of pregnancy and to have a large sample size. We chose to initiate the fetal renal artery Doppler assessment in the early second trimester because the fetal renal pathologic presentation starts at this time, so reference data on the normal renal artery is required [10]. However, awareness about Doppler assessment in small vessels is important so in our study the sample volume was reduced to be appropriate for the fetal renal artery, which was shown by color Doppler.

Our study found 3 patterns of renal artery Doppler waveform. Most of them were type III. This finding is similar to the previous studies [4, 9], which found that all 3 patterns of renal artery Doppler can be seen among normal fetuses, although the percentages of such patterns differed from those in our study. An explanation may lie in the differences in gestational age in each study and the small sample size in previous studies. Most of cases in previous studies were fetuses in the third trimester of pregnancy $[4,9]$. Interestingly, we found that during the second trimester of pregnancy the fetal artery Doppler indices, including of PI, PSV, S/D and RI (with AEDV removed), each increased significantly with gestational age, but the increase in EDV (with AEDV removed) with gestational age was not significant. We postulate that the increase in fetal renal artery PSV was related to the increase in renal blood flow and fetal urine production rate with advancing gestation [11]. Although, the PI, RI, and S/ $D$ increased with advancing gestational age, the values were not high enough to interrupt the renal perfusion. Finally, we constructed best-fitting regression equations and reference ranges for each Doppler index.

The study population included pregnant women between 14 and 28 weeks who mostly resided in urban areas of southern Thai area. Although our participants were mostly Muslim, other basic demographic data including economic status was similar for that of health care in other sites in Thailand. Thus, it should be appropriate to extrapolate the normal reference range for the fetal renal artery to all pregnant Thai women.

In conclusion, these normative data could serve as a basis for evaluation of the fetal renal artery blood flow, which should be of benefit for further pregnancy management, especially in high risk situations that interfere with fetal renal perfusion.

\section{Acknowledgements}

The authors would like to thank Mr David Patterson from the International Affairs Office, Faculty of Medicine, Prince of Songkla University, Songkhla, Thailand, for editorial assistance. This article has been presented as an oral presentation at the 23rd Asian and Oceanic Congress of Obstetrics and Gynaecology, October 20-23, 2013, Bangkok, Thailand.

The authors report no conflict of interest. The authors alone are responsible for the content and writing of the paper.

\section{References}

1. Azpurua H, Dulay AT, Buhimschi IA, Bahtiyar MO, Funai E, Abdel-Razeq SS, et al. Fetal renal artery impedance as assessed by Doppler ultrasound in pregnancies complicated by intraamniotic inflammation and preterm birth. Am J Obstet Gynecol. 2009; 200: 203e1-11.

2. Stigter RH, Mulder EJ, Bruinse HW, Visser GH. Doppler studies on the fetal renal artery in the severely growth-restricted fetus. Ultrasound Obstet Gynecol. 2001; 18:141-5.

3. Vyas S, Nicolaides KH, Campbell S. Renal artery flow-velocity waveforms in normal and hypoxemic fetuses. Am J Obstet Gynecol. 1989; 161:168-72.

4. Hecher K, Spernol R, Szalay S. Doppler blood flow velocity waveforms in the fetal renal artery. Arch Gynecol Obstet. 1989; 246:133-7.

5. Haugen G, Godfrey K, Crozier S, Hanson M. Doppler blood flow velocity waveforms in the fetal renal arteries: variability at proximal and distal sites in the right and left arteries. Ultrasound Obstet Gynecol. 2004; 23:590-3.

6. Tanabe R. Doppler ultrasonographic assessment of fetal renal artery blood flow velocity waveforms in intrauterine growth retarded fetuses. Kurume Med J. 1992; 39:203-8.

7. Konje JC, Abrams KR, Taylor DJ. Normative values of Doppler velocimetry of five major fetal arteries as determined by color power angiography. Acta Obstet 
Gynecol Scand. 2005; 84:230-7.

8. Veille JC, Kanaan C. Duplex Doppler ultrasonographic evaluation of the fetal renal artery in normal and abnormal fetuses. Am J Obstet Gynecol. 1989; 161: 1502-7.

9. Iura T, Makinoda S, Fujita S, Matsuzawa S, Waseda T, Ohshima K, et al. Analysis of renal artery hemodynamics in normal fetuses using the color Doppler method. Fetal Diagn Ther. 2005; 20:86-90.

10. Winyard P, Chitty LS. Dysplastic kidney. Semin Fetal Neonatal Med. 2008; 13:142-51.

11. Cambell S, Wladimiroff JW, Dewhurst CJ. The antenatal measurement of fetal urine production. J Obstet Gynaecol Br Commonw. 1973; 80:680-6. 